\title{
Sun-Synchronous Highly Elliptical Orbits using Low-Thrust Propulsion
}

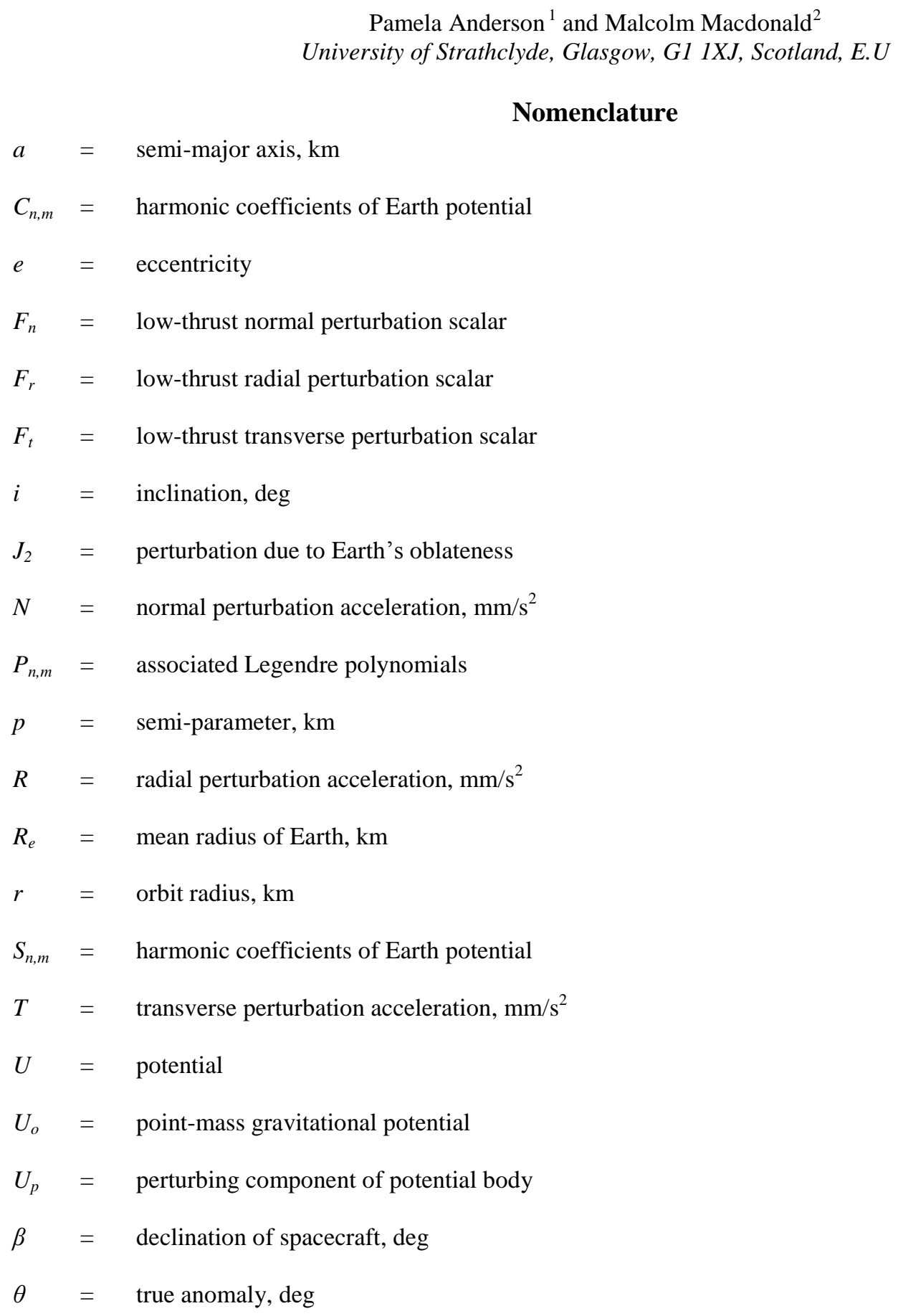

Nomenclature

$a=$ semi-major axis, $\mathrm{km}$

$C_{n, m}=$ harmonic coefficients of Earth potential

$e \quad=$ eccentricity

$F_{n} \quad=\quad$ low-thrust normal perturbation scalar

$F_{r}=$ low-thrust radial perturbation scalar

$F_{t}=$ low-thrust transverse perturbation scalar

$i=$ inclination, deg

$J_{2}=$ perturbation due to Earth's oblateness

$N=$ normal perturbation acceleration, $\mathrm{mm} / \mathrm{s}^{2}$

$P_{n, m}=$ associated Legendre polynomials

$p \quad=\quad$ semi-parameter, $\mathrm{km}$

$R=$ radial perturbation acceleration, $\mathrm{mm} / \mathrm{s}^{2}$

$R_{e} \quad=\quad$ mean radius of Earth, $\mathrm{km}$

$r \quad=\quad$ orbit radius, $\mathrm{km}$

$S_{n, m}=$ harmonic coefficients of Earth potential

$T=$ transverse perturbation acceleration, $\mathrm{mm} / \mathrm{s}^{2}$

$U=$ potential

$U_{o}=$ point-mass gravitational potential

$U_{p} \quad=\quad$ perturbing component of potential body

$\beta=$ declination of spacecraft, deg

$\theta=$ true anomaly, deg

${ }^{1} \mathrm{PhD}$ Candidate, Advanced Space Concepts Laboratory, University of Strathclyde, pamela.c.anderson@strath.ac.uk. ${ }^{2}$ Associate Director, Advanced Space Concepts Laboratory, University of Strathclyde, malcolm.macdonald.102@strath.ac.uk, Associate Fellow of the AIAA.

American Institute of Aeronautics and Astronautics 
$\lambda=$ geographical longitude, $\operatorname{deg}$

$\mu \quad=\quad$ gravitational parameter of Earth, $\mathrm{km}^{3} / \mathrm{s}^{2}$

$\Omega \quad=\quad$ ascending node angle, $\mathrm{deg}$

$\omega=$ argument of perigee, $\mathrm{deg}$

\section{Introduction}

$\mathrm{D}$ UE to restrictions within the current architecture of the global observing system (GOS), space-based remote sensing of Earth suffers from an acute data-deficit over the critical polar-regions. Currently, observation of high-latitude regions is conducted using composite images from spacecraft in geostationary (GEO) and low-Earth orbits (LEOs) [1]. However, the oblique viewing geometry from GEO-based systems to latitudes above around 55 deg [2] and the insufficient temporal resolution of spacecraft in LEO means there is currently no source of continuous imagery for polar-regions obtained with a data refresh rate of less than 15 minutes, as is typically available elsewhere for meteorological observations.

The use of high inclination orbits for improved polar monitoring has recently been endorsed by the World Meteorological Organization [3], stating that a Highly Elliptical Orbit (HEO) constellation is required, complementing the fleet of GEO satellites achieving continuous global coverage. One example of a HEO is the Molniya orbit, which has a period of $12 \mathrm{hrs}$ and "critical-inclination" of $63.43 \mathrm{deg}$ or $116.6 \mathrm{deg}$ at either of these inclinations there is no change in the argument of perigee of the orbit, usually caused by the Earth's equatorial bulge. Although this offers an alternative for polar remote sensing, the inclination means a single platform on a Molniya orbit cannot provide hemispheric observations to the latitudes required to overcome the identified data deficit (55 deg latitude). As a result, observations would continue to be dependent upon composite images. To provide continuous observation to the latitudes required, with composite images, three spacecraft on Molniya orbits in three separate orbit planes are required.

Newly proposed HEOs, termed Taranis orbits, use continuous acceleration to modify the magnitude of the perturbing acceleration from a Keplerian orbit allowing free selection of the critical inclination to any value required by the mission. Taranis orbits have been introduced by Anderson and Macdonald [4, 5], where the method for determining the acceleration required to achieve orbits with varying inclinations is detailed, for both solar electric 
propulsion (SEP) and hybrid solar-sail/SEP systems respectively. Previous work also pays particular attention to a $12 \mathrm{~h}$ Taranis orbit with apogee altitude similar to that of a Molniya orbit and an inclination of $90 \mathrm{deg}$. The authors have also considered the design of a constellation of spacecraft on Taranis orbits to give continuous observation above 55 deg latitude. Only two spacecraft, in a single orbit plane, for a $12 \mathrm{~h}$ orbit are required to provide continuous observation using composite images. However, a third spacecraft in the same orbit plane enables continuous visibility without the use of composite images. Critically, the use of a single orbit plane significantly reduces the constellation deployment costs.

This paper builds upon the work introduced in [4-6] and develops sun-synchronous Taranis orbits, using methods detailed in recent research for the extension of sun-synchronous orbits [7]. Within this work, the thrust magnitude required is not defined as a function of the local gravity field but instead by the magnitude of the perturbations within that field, augmenting the Earth oblateness perturbation to modify the sun-synchronous orbit and allow free selection of the orbit inclination and altitude. Previously, Taranis orbits have been developed using continuous radial and transverse accelerations, thus sun-synchronous Taranis orbits are achieved by the addition of a further element of acceleration, directed out of the orbit plane. This ensures the rate of change of the ascending node of the Taranis orbit matches the mean rotation of the sun within an Earth-centered inertial (ECI) frame. It is likely that sun-synchronous HEOs will offer benefits in terms of simplified instrument design through simplification of the thermal environment.

\section{Spacecraft Motion about an Oblate Body with Low-Thrust Propulsion}

\section{A. General Perturbations Solution}

A sun-synchronous orbit requires that the rate of change of the ascending node matches the motion of the mean sun ( $2 \pi$ radians in 365.25 days). The ascending node can be described within an ECI reference frame through the Gaussian form of the variational equations of the classical orbital elements [8]

$$
\frac{d \Omega}{d \theta}=\frac{r^{3}}{\mu p \sin i} \sin (\theta+\omega) N
$$

Taranis orbits also require the rate of change of argument of perigee to remain unchanged in order that the position of apogee is not affected by the perturbations caused by the oblate nature of the Earth. The rate of change of argument of perigee is given as [8] 


$$
\frac{d \omega}{d \theta}=\frac{r^{2}}{\mu e}\left[-R \cos \theta+T\left(1+\frac{r}{p}\right) \sin \theta\right]-\frac{r^{3}}{\mu p \tan i} \sin (\theta+\omega) N
$$

The disturbing force components in the radial, transverse and normal (RTN) directions are given by

$$
\begin{aligned}
& R_{J_{2}+F_{r}}=\frac{3}{2} \frac{J_{2} \mu R_{e}^{2}}{r^{4}}\left(3 \sin ^{2}(i) \sin ^{2}(\theta+\omega)-1\right)+F_{r} \operatorname{sgn}[\cos \theta] \\
& T_{J_{2}+F_{t}}=-\frac{3}{2} \frac{J_{2} \mu R_{e}^{2}}{r^{4}} \sin ^{2}(i) \sin 2(\theta+\omega)+F_{t} \operatorname{sgn}[\sin \theta] \\
& N_{J_{2}+F_{n}}=-\frac{3}{2} \frac{J_{2} \mu R_{e}^{2}}{r^{4}} \sin (2 i) \sin (\theta+\omega)+F_{n} \operatorname{sgn}[\sin (\theta+\omega)]
\end{aligned}
$$

These expressions contain contributions from the Earth oblateness effect, $J_{2}$, and acceleration terms, which are added to the perturbations using locally optimal control laws, which maximize the instantaneous rate of change of the orbital element and provide the thrust orientation in analytical form [9]. The control laws also give the distinct position on the orbit where the sign of the thrust is required to switch direction.

\section{Ascending Node Angle}

A continuous acceleration is firstly added to the out of plane perturbation to ensure the change in ascending node angle is equal to approximately 1 deg per day for any orbit under consideration and maintain the sun-synchronous condition. The expression for the rate of change of ascending node with the application of low-thrust is determined by inserting Eq. (5) into Eq. (1)

$$
\frac{d \Omega}{d \theta}=\frac{a^{2}\left(1-e^{2}\right)^{2} \sin (\omega+\theta)}{\sin (i) \mu(1+e \cos (\theta))^{3}}\left(F_{n} \operatorname{sgn}[\sin (\theta+\omega)]-\frac{3 J_{2} R_{e}^{2} \mu(1+e \cos (\theta))^{4} \sin (2 i) \sin (\omega+\theta)}{2 a^{4}\left(1-e^{2}\right)^{4}}\right)
$$

It is noted that the normal low-thrust component switches sign as a function of argument of latitude; consequently, the value assigned to the argument of perigee becomes important. Thus, considering argument of perigee equal to both 0 deg and 270 deg and integrating Eq. (6) over one orbital revolution results in two expressions for the change in ascending node angle 


$$
\begin{aligned}
(\Delta \Omega)_{0}^{2 \pi} & =\left(-\frac{3 J_{2} R_{e}^{2} \cos (i)}{a^{2}\left(-1+e^{2}\right)^{2}}\right)+\left(\frac{4 a^{2} F_{n} \cos (\omega)}{\mu \sin (i)}\right) \\
(\Delta \Omega)_{0}^{2 \pi} & =\left(-\frac{1}{\sin (i) \sqrt{-1+e^{2}} \mu} a^{2} F_{n}\left(4 \sqrt{-1+e^{2}}+2 e^{2} \sqrt{-1+e^{2}}-12 e \operatorname{ArcTanh}\left[\frac{-1+e}{\sqrt{-1+e^{2}}}\right]-3 e \ln \left[\frac{1-e}{\sqrt{-1+e^{2}}}\right]+3 e \ln \left[\frac{-1+e}{\sqrt{-1+e^{2}}}\right]\right) \sin (\omega)\right) \\
& +\left(-\frac{3 J_{2} \pi R_{e}^{2} \cos (i)}{a^{2}\left(-1+e^{2}\right)^{2}}\right)
\end{aligned}
$$

Switching the rate of change of ascending node per rotation to per second gives

$$
\begin{aligned}
& \Delta \Omega=\frac{1}{2 \pi} \sqrt{\frac{\mu}{a^{3}}}\left(-\frac{3 \pi J_{2} R_{e}^{2} \cos (i)}{a^{2}\left(-1+e^{2}\right)^{2}}+\frac{4 a^{2} F_{n} \cos (\omega)}{\mu \sin (i)}\right) \\
& \Delta \Omega=\frac{1}{2 \pi} \sqrt{\frac{\mu}{a^{3}}}\left(\begin{array}{l}
-\frac{3 J_{2} \pi R_{e}^{2} \cos (i)}{a^{2}\left(-1+e^{2}\right)^{2}}-1 / \\
\left(\sqrt{-1+e^{2}} \mu\right) a^{2} F_{n} \csc (i) \\
\left(4 \sqrt{-1+e^{2}}+2 e^{2} \sqrt{-1+e^{2}}-12 e \operatorname{ArcTanh}\left[\frac{-1+e}{\sqrt{-1+e^{2}}}\right]-3 e \ln \left[\frac{1-e}{\sqrt{-1+e^{2}}}\right]+3 e \ln \left[\frac{-1+e}{\sqrt{-1+e^{2}}}\right]\right) \sin (\omega)
\end{array}\right)
\end{aligned}
$$

If no continuous acceleration is applied, Eqs (9) and (10) simplify to the standard expression for the sunsynchronous orbit

$$
i=\cos ^{-1}\left[-\frac{2}{3} \frac{\Delta \Omega}{J_{2}} \frac{a^{7 / 2}\left(1-e^{2}\right)^{2}}{R_{e}^{2} \sqrt{\mu}}\right]
$$

\section{Argument of Perigee}

Sun-synchronous Taranis orbits are also required to give zero change in the argument of perigee over one orbital revolution. Thus, continuous radial and transverse accelerations are added to maintain this condition and compensate for the applied out-of-plane acceleration. The rate of change of argument of perigee is found by substituting Eqs. (3) - (5) into Eq. (2) to give 


$$
\begin{aligned}
\frac{d \omega}{d \theta}= & -\frac{a^{2}\left(1-e^{2}\right)^{2} \cot (i) \sin (\theta+\omega)\left(F_{n} \operatorname{sgn}[\sin (\theta+\omega)]-\frac{3 J_{2} R_{e}^{2} \mu(1+e \cos \theta)^{4} \sin (2 i) \sin (\theta+\omega)}{2 a^{4}\left(1-e^{2}\right)^{4}}\right)}{\mu(1+e \cos \theta)^{3}} \\
& +\frac{1}{e \mu(1+e \cos \theta)^{2}} a^{2}\left(1-e^{2}\right)^{2}\left(\cos \theta\left(-F_{r} \operatorname{sgn}[\cos \theta]-\frac{3 J_{2} R_{e}^{2} \mu(1+e \cos \theta)^{4}\left(-1+3 \sin ^{2}(i) \sin ^{2}(\theta+\omega)\right)}{2 a^{4}\left(1-e^{2}\right)^{4}}\right)\right) \\
& +\left(1+\frac{1}{1+e \cos \theta}\right) \sin \theta\left(F_{t} \operatorname{sgn}[\sin \theta]-\frac{3 J_{2} R_{e}^{2} \mu(1+e \cos (\theta))^{4} \sin ^{2}(i) \sin 2(\theta+\omega)}{2 a^{4}\left(1-e^{2}\right)^{4}}\right)
\end{aligned}
$$

The change in argument of perigee is found by integrating Eq. (11) over one orbital revolution. The total change in argument of perigee is made up of four terms, consisting of the change in argument of perigee due to $J_{2}$ effects and due to the effects of each of the $R, T$, and $N$ accelerations.

$$
(\Delta \omega)_{0}^{2 \pi}=(\Delta \omega)_{J_{2}}+(\Delta \omega)_{F_{r}}+(\Delta \omega)_{F_{t}}+(\Delta \omega)_{F_{n}}
$$

The acceleration due to $J_{2}$ is given by

$$
(\Delta \omega)_{J_{2}}=\frac{3 J_{2} \pi R_{e}^{2}(3+5 \cos (2 i))}{4 a^{2}\left(-1+e^{2}\right)}
$$

To determine the natural critical inclination, Eq. (12) containing only the effects of $J_{2}$, from Eq (13), is set to zero resulting in inclinations of $63.43 \mathrm{deg}$ and $116.6 \mathrm{deg}$. Thus, all Earth orbits inclined at these values show no rotation of the apsidal line, irrespective of the semi-major axis or eccentricity. However, these fixed critical inclinations place restrictions on design and thus limit the applications of Molniya-like orbits for high-latitude observation. It is evident from Eq. (12) that altering the inclination from these values will result in a drift in the argument of perigee due to the effect of $J_{2}$ perturbations. Thus, for each value of inclination there exists a constant acceleration magnitude which will negate this drift, and allow free selection of inclination. Acceleration components in the radial, transverse and normal directions are given by

$$
(\Delta \omega)_{F_{r}}=\frac{1}{e \mu} 2 a^{2} F_{r}\left(-2+2 e^{2}-4 e \sqrt{-1+e^{2}} \operatorname{Arctanh}\left[\frac{-1+e}{\sqrt{-1+e^{2}}}\right]-e \sqrt{-1+e^{2}} \ln \left[\frac{1-e}{\sqrt{-1+e^{2}}}\right]+e \sqrt{-1+e^{2}} \ln \left[\frac{-1+e}{\sqrt{-1+e^{2}}}\right]\right)
$$




$$
(\Delta \omega)_{F_{t}}=-\frac{4 a^{2}\left(-2+e^{2}\right) F_{t}}{e \mu}
$$

Once again, the normal low-thrust term switches sign as a function of the argument of latitude. Thus, two solutions exist for argument of perigee equal to 0 deg and $270 \mathrm{deg}$.

$$
\begin{aligned}
& (\Delta \omega)_{F_{n}}^{\omega=0}=-\frac{4 a^{2} F_{n} \cos (\omega) \cot (i)}{\mu} \\
& (\Delta \omega)_{F_{n}}^{\omega=\frac{\pi}{2}}=\frac{1}{\sqrt{-1+e^{2}} \mu} a^{2} F_{n} \cot (i)\left(\begin{array}{l}
\left.4 \sqrt{-1+e^{2}}+2 e^{2} \sqrt{-1+e^{2}}-12 e \operatorname{Arctanh}\left[\frac{-1+e}{\sqrt{-1+e^{2}}}\right]\right) \sin (\omega) \\
-3 e \ln \left[\frac{1-e}{\sqrt{-1+e^{2}}}\right]+3 e \ln \left[\frac{-1+e}{\sqrt{-1+e^{2}}}\right]
\end{array}\right)
\end{aligned}
$$

Although two solutions exist depending on the value of argument of perigee, an argument of perigee of $270 \mathrm{deg}$ is considered in the remainder of the paper.

Equation (10) is firstly considered by substituting values of the orbital elements and solving for the continuous acceleration, $F_{n}$. This is substituted, along with the orbital element values into Eq. (12), which is solved for the required radial and transverse accelerations, to compensate for the applied out of plane acceleration and maintain the zero change in argument of perigee condition. The total acceleration magnitude to achieve sun-synchronous Taranis orbits of varying orbital period and inclination are given in Fig. 1, where the perigee altitude is $813 \mathrm{~km}$. 


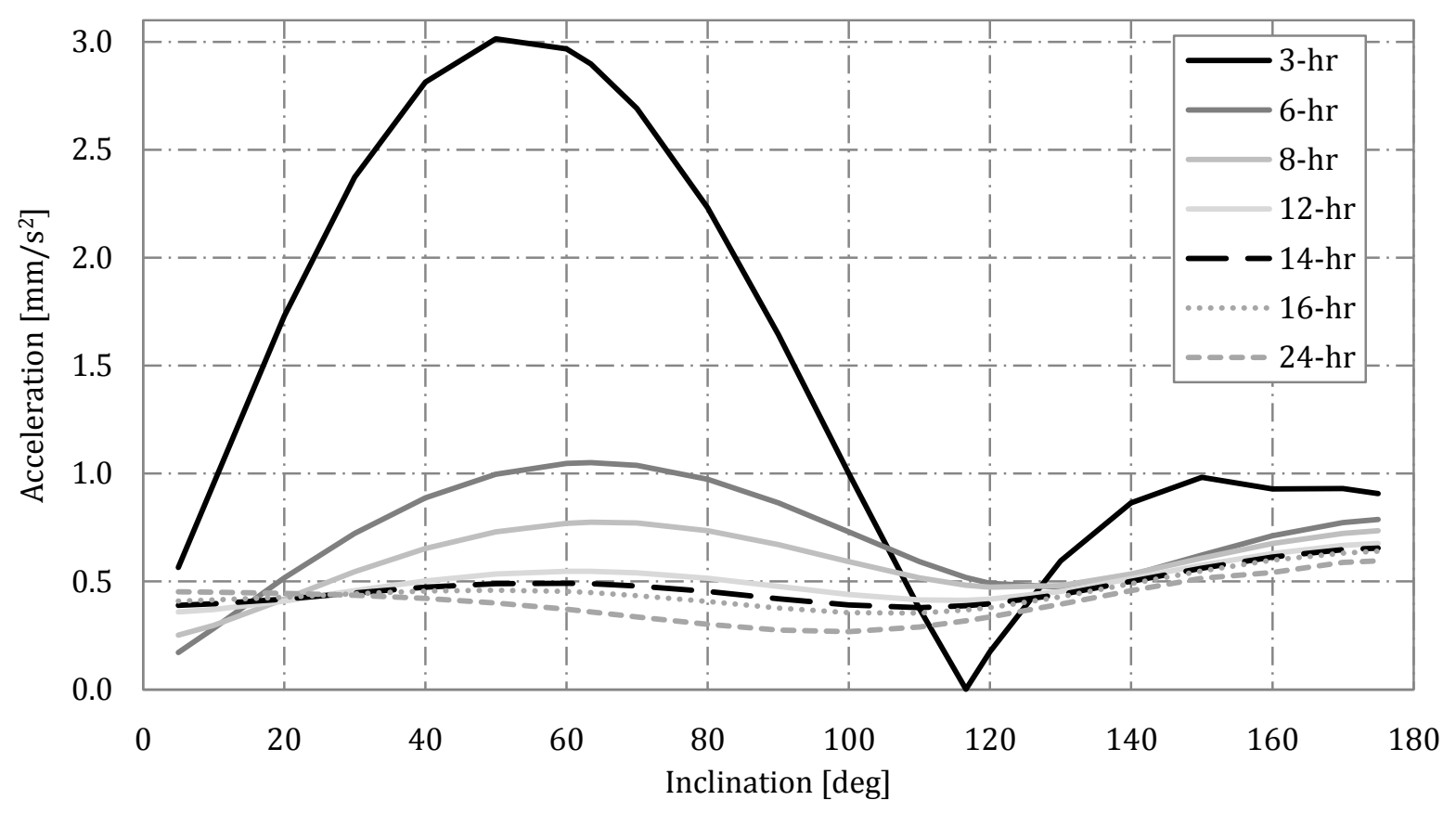

Fig. 1 Total acceleration magnitude to achieve sun-synchronous Taranis orbits of varying period and inclination.

Fig. 1 shows that a natural sun-synchronous orbit exists without the use of any low-thrust propulsion. This occurs for a $3 \mathrm{~h}$ orbit at the conventional critical inclination of $116.6 \mathrm{deg}$. As the perigee altitude remains constant for all orbits in Fig. 1, only one natural sun-synchronous exists i.e. the $3 \mathrm{~h}$ orbit. Thus, the other results shown in Fig. 1 are an extension of this solution, and are only enabled through the use of continuous acceleration. For improved high-latitude observation the most beneficial inclination is $90 \mathrm{deg}$ in the case of a $12 \mathrm{~h}$ orbit, the orbit considered in previous publications [4, 5], the total constant acceleration magnitude required is $0.478 \mathrm{~mm} / \mathrm{s}^{2}$. In this case, the majority of the acceleration is in the normal direction $\left(0.470 \mathrm{~mm} / \mathrm{s}^{2}\right)$. Thus, for a 1-ton spacecraft a maximum thrust of $478 \mathrm{mN}$ is required, which will decrease as the propellant is consumed a variable thrust SEP system is therefore required Although the maximum thrust is higher than the thrust required to achieve a 90 deg inclination Taranis orbit without the sun-synchronous condition $(81 \mathrm{mN})$ this level of thrust is achievable using current or near-term technology. For example, the High Power Electric Propulsion thruster which has undergone ground testing is capable of providing a maximum of $670 \mathrm{mN}$, [10] and test data from the Nuclear Electric Xenon Ion System has also shown a maximum thrust level of $476 \mathrm{mN}$ [11]. 
Fig. 1 also shows that as the orbital period increases, the acceleration magnitude decreases. To reach an inclination of $90 \mathrm{deg}$ a $24 \mathrm{~h}$ orbit requires a constant acceleration of $0.275 \mathrm{~mm} / \mathrm{s}^{2}$, while a $6 \mathrm{~h}$ orbit requires a constant acceleration magnitude of $0.865 \mathrm{~mm} / \mathrm{s}^{2}$. Thus, lower orbit periods are expected to require development in SEP technology before they become feasible.

\section{B. Change in Orbital Elements}

Analytical solutions are developed for the remaining orbital elements, to ensure the desired zero secular rate of change of orbital elements is maintained in the presence of continuous acceleration.

1. Semi-Major Axis

$$
\frac{d a}{d \theta}=\frac{2 p r^{2}}{\mu\left(1-e^{2}\right)^{2}}\left(R_{J_{2}+F_{r}} e \sin \theta+T_{J_{2}+F_{t}} \frac{p}{r}\right)
$$

Substituting expressions for the perturbing accelerations gives

$$
\begin{aligned}
\frac{d a}{d \theta}= & \frac{1}{\mu(1+e \cos \theta)^{2}} 2 a^{3}\left(1-e^{2}\right)\left(e \sin \theta\left(F_{r}+\frac{3 J_{2} R_{e}^{2} \mu(1+e \cos \theta)^{4}\left(-1+3 \sin ^{2}(i) \sin ^{2}(\theta+\omega)\right)}{2 a^{4}\left(1-e^{2}\right)^{4}}\right)\right) \\
& +(1+e \cos \theta)\left(F_{t}-\frac{3 J_{2} R_{e}^{2} \mu(1+e \cos \theta)^{4} \sin ^{2}(i) \sin 2(\theta+\omega)}{2 a^{4}\left(1-e^{2}\right)^{4}}\right)
\end{aligned}
$$

Integrating Eq. (19) over one revolution to give the change in semi-major axis as

$$
(\Delta a)_{0}^{2 \pi}=0
$$

\section{Eccentricity}

$$
\frac{d e}{d \theta}=\frac{r^{2}}{\mu p}\left(R_{J_{2}+F_{r}} \sin \theta+T_{J_{2}+F_{r}}\left(\left(1+\frac{r}{p}\right) \cos \theta+\frac{r e}{p}\right)\right)
$$

Inserting the perturbations into Eq. (21) gives 


$$
\begin{aligned}
\frac{d e}{d \theta}= & \frac{1}{\mu(1+e \cos \theta)^{2}} a\left(1-e^{2}\right)\left(\sin \theta\left(F_{r}+\frac{3 J_{2} R_{e}^{2} \mu(1+e \cos \theta)^{4}\left(-1+3 \sin ^{2}(i) \sin ^{2}(\theta+\omega)\right)}{2 a^{4}\left(1-e^{2}\right)^{4}}\right)\right) \\
& +\left(\frac{e}{1+e \cos \theta}+\cos \theta\left(1+\frac{1}{1+e \cos \theta}\right)\right)\left(F_{t}-\frac{3 J_{2} R_{e}^{2} \mu(1+e \cos \theta)^{4} \sin (i)^{2} \sin 2(\theta+\omega)}{2 a^{4}\left(1-e^{2}\right)^{4}}\right)
\end{aligned}
$$

Integrating over one revolution, the change in eccentricity is

$(\Delta e)_{0}^{2 \pi}=0$

\section{Inclination}

$$
\frac{d i}{d \theta}=\frac{r^{3}}{\mu p} \cos (\theta+\omega) N_{J_{2}+F_{n}}
$$

Substituting the normal perturbation, Eq. (24) becomes

$$
\frac{d i}{d \theta}=\frac{a^{2}\left(1-e^{2}\right)^{2} \cos (\theta+\omega)}{\mu(1+e \cos \theta)^{3}}\left(F_{n}-\frac{3 J_{2} R_{e}^{2} \mu(1+e \cos \theta)^{4} \sin (2 i) \sin (\theta+\omega)}{2 a^{4}\left(1-e^{2}\right)^{4}}\right)
$$

Once more, the locally optimal control law states that the normal thrust switches sign depending on argument of latitude; consequently Eq. (25) is integrated over one orbit using both argument of perigee equal to 0 deg and 270 deg gives the change in inclination respectively

$$
\begin{aligned}
& (\Delta i)_{0}^{2 \pi}=\left[-\frac{4 a^{2} F_{n} \sin (\omega)}{\mu}\right]_{\omega=0 \mathrm{deg}} \\
& (\Delta i)_{0}^{2 \pi}=\left[-\frac{1}{\sqrt{-1+e^{2}} \mu} a^{2} F_{n} \cos (\omega)\left(4 \sqrt{-1+e^{2}}+2 e^{2} \sqrt{-1+e^{2}}-12 e \operatorname{Arctanh}\left[\frac{-1+e}{\sqrt{-1+e^{2}}}\right]-3 e \ln \left[\frac{1-e}{\sqrt{-1+e^{2}}}\right]+3 e \ln \left[\frac{-1+e}{\sqrt{-1+e^{2}}}\right]\right)\right]_{\omega=270 \operatorname{deg}}
\end{aligned}
$$

Inserting values of orbital elements gives the change in inclination for both cases as

$$
(\Delta i)_{0}^{2 \pi}=0
$$


Thus, these analytical solutions show no adverse affect on any of the orbital elements through the application of continuous low-thrust.

\section{Special Perturbations Technique}

To verify the solutions generated using the general perturbations method, a special perturbations solution is generated. This numerical model propagates the position of the spacecraft,with initial conditions given in Table 1, using a set of Modified Equinoctial Elements [12], using an explicit variable step size Runge Kutta $(4,5)$ formula, the Dormand-Prince pair [13]. Numerical simulations include only perturbations due to Earth oblateness to the order of $J_{2}$ and relative and absolute error tolerances are set to $10^{-8}$. The acceleration control profiles, given from the locally optimal control laws, to maintain the 12 h, 90 deg incliantion Taranis orbit are given in Fig. 2 - Fig. 4.

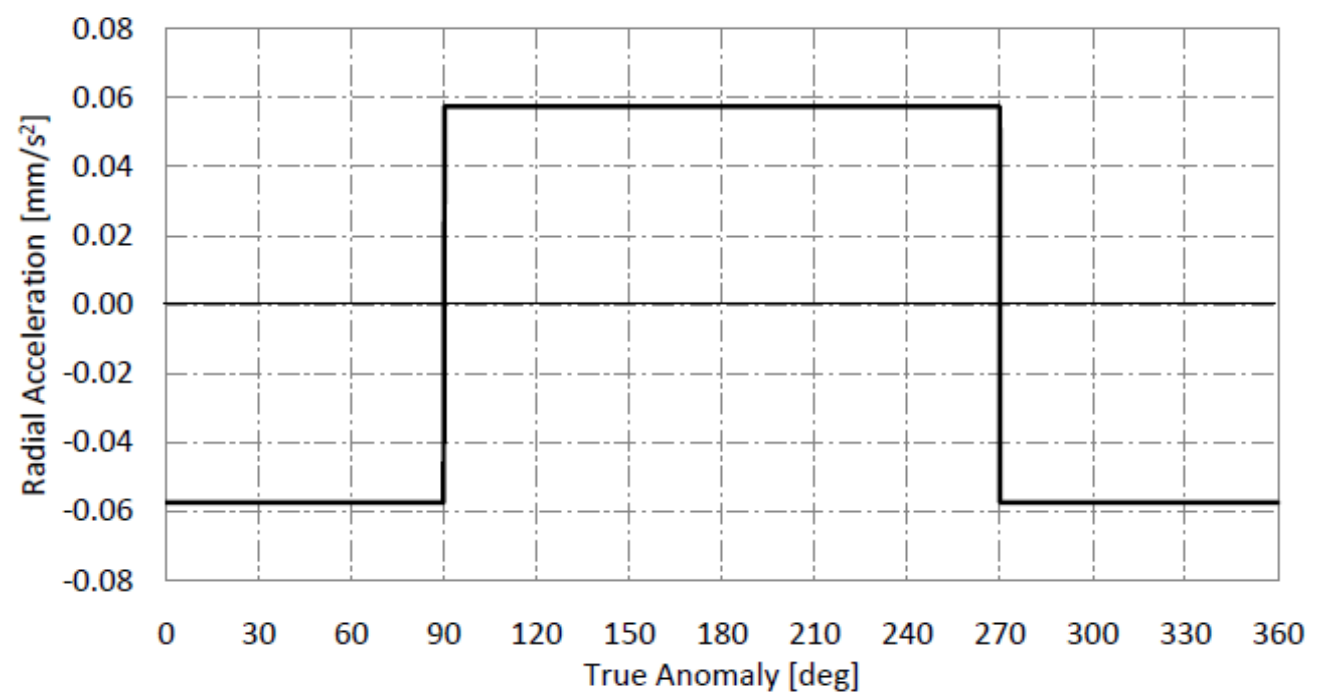

Fig. 2 Radial control profile over one orbital revolution. 


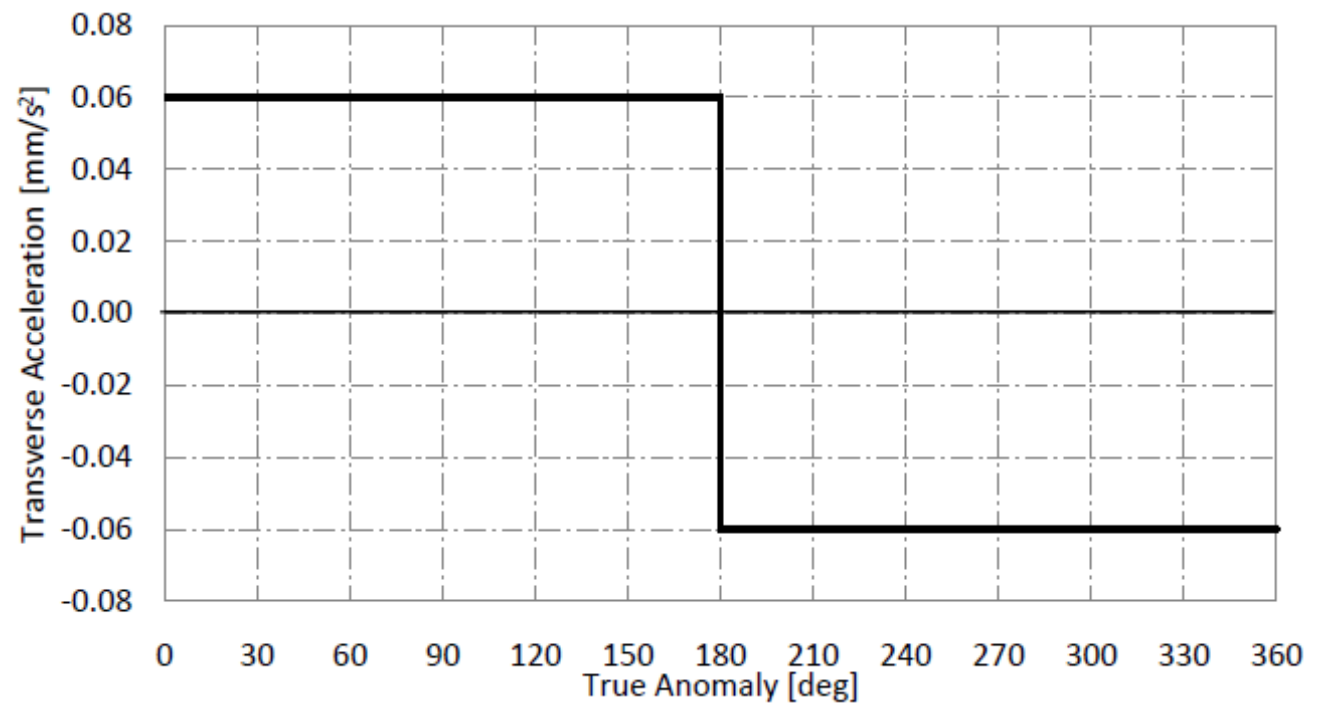

Fig. 3 Transverse control profile over one orbital revolution.

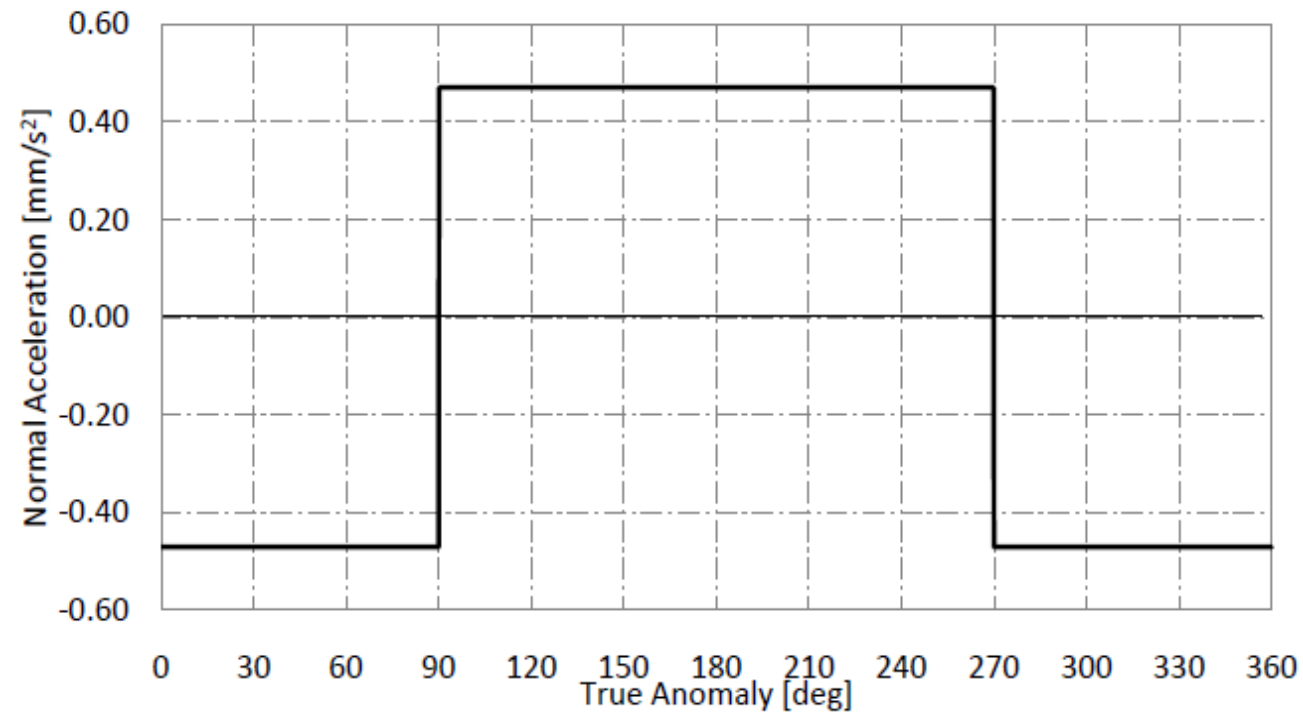

Fig. 4 Normal control profile over one orbital revolution.

It can be seen from Fig. 2 - Fig. 4 the relative simplictity of the control profiles implemented to enable the $12 \mathrm{~h}$, 90 deg Taranis orbit. Although the controls used are not globally optimal, previous studies on the $12 \mathrm{~h}$ Taranis orbit without the sun-synchronous condition have performed optimization using PSOPT, which was shown to produce a propellant saving of $<4 \%$, this mass saving was deemed to be negligible due to the significant increase in complexity of the control profiles in this case [4]. Optimisation of the controls has therefore not been performed for the sun-synchronous Taranis orbits presented in this paper. Although the results shown in this paper are for continuous, constant acceleration as the propellant is consumed and the spacecraft mass decreases thrust arcs could 
be introduced into the orbit, thus increasing the tolerances to thrust failure significant investigation of this is however, out with the sope of this work.

The numerical model verifies that the change in argument of perigee is negligible over one orbit revolution, and the change in ascending node angle is approximately equal to 1 deg per day, this is shown in Fig. 5 and Fig. 6 respectively. The numerical model also confirms that the change in semi-major axis, eccentricity and inclination are negligible over one orbit revolution. The change in semi-major axis, eccentricity and inclination over five orbital revolutions are shown in Fig. 7.

Table 1 Orbital parameters.

\begin{tabular}{lc}
\hline \hline & \\
Orbital Element & Value \\
\hline Perigee Altitude & $813(\mathrm{~km})$ \\
Apogee Altitude & $39540(\mathrm{~km})$ \\
Ascending Node & $330(\mathrm{deg})$ \\
Argument of perigee & $270(\mathrm{deg})$ \\
\hline \hline
\end{tabular}

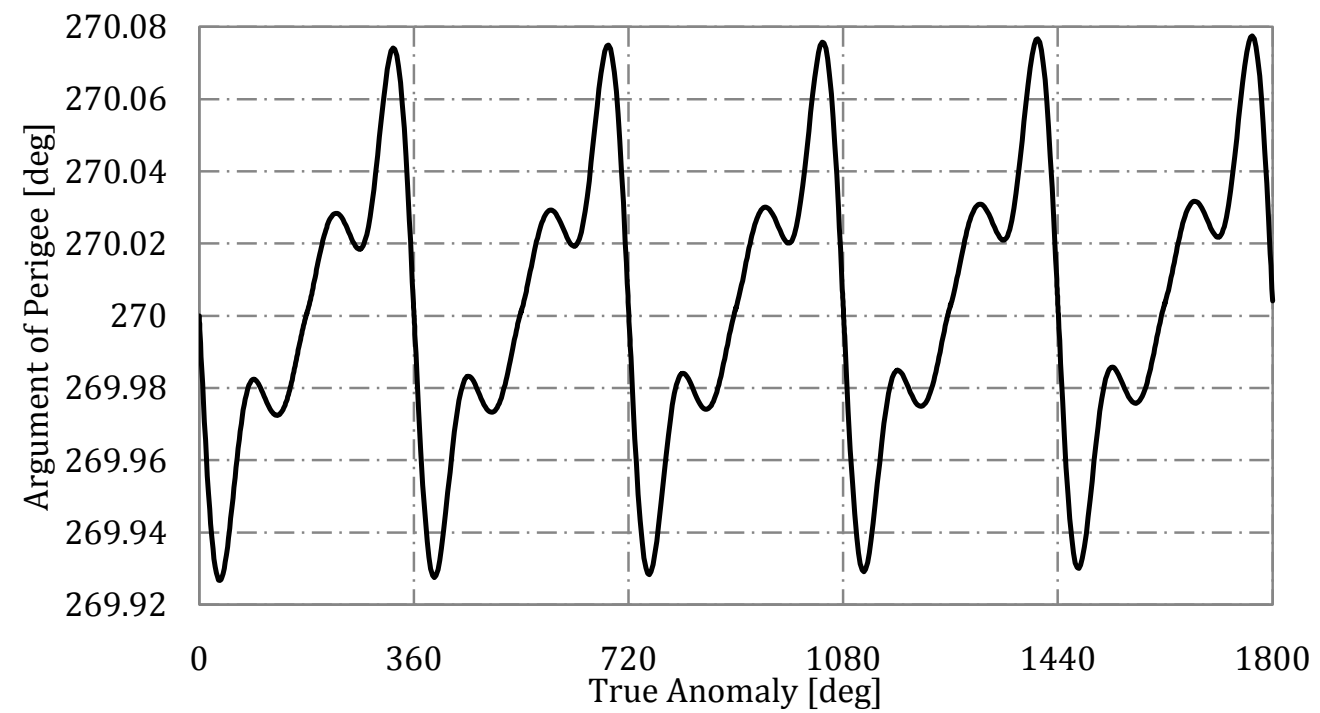

Fig. 5 Varaition of argument of perigee over five orbital revolutions. 


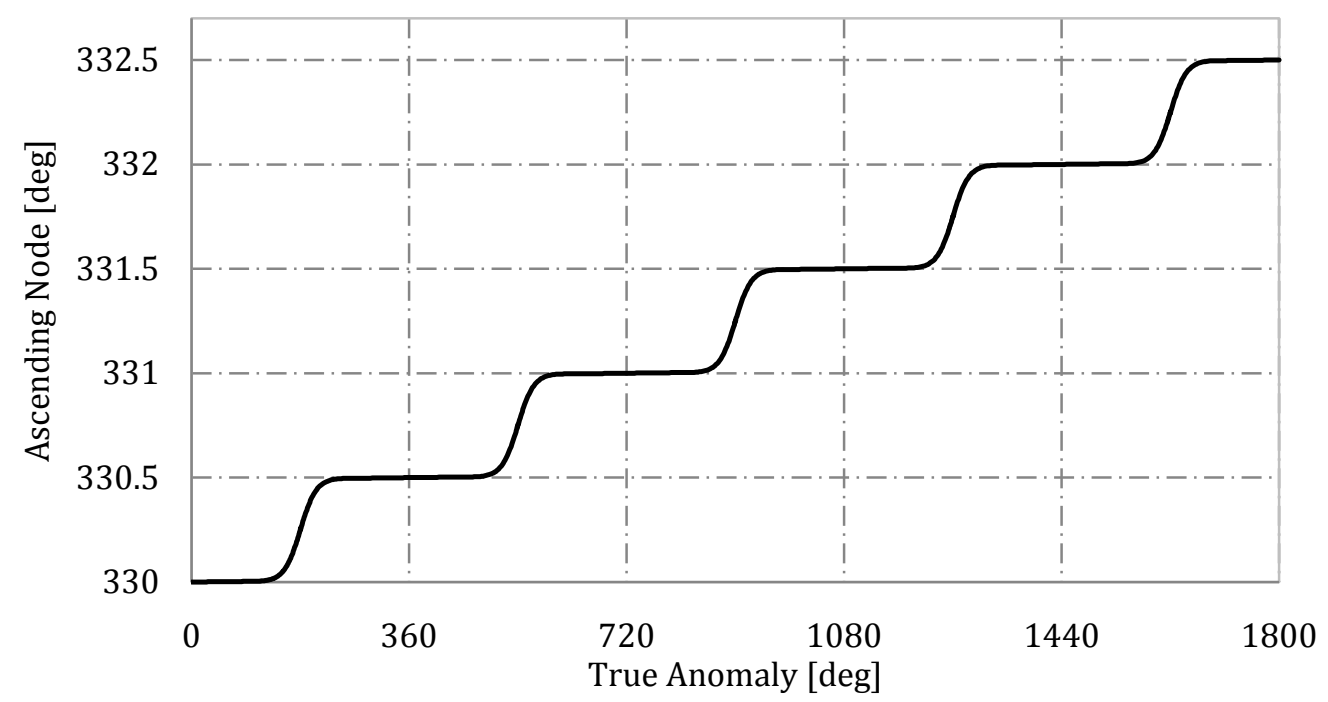

Fig. 6 Variation of longitude of ascending node over five orbital revolutions.

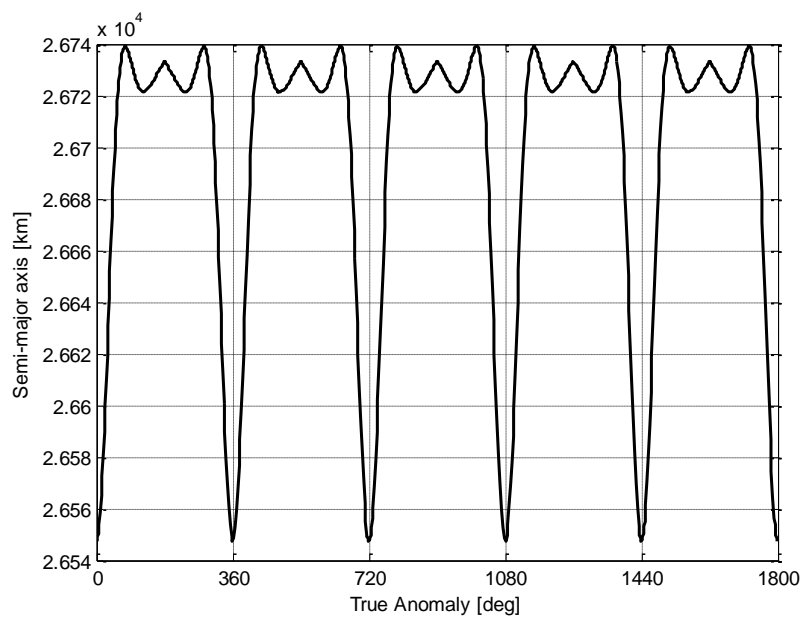

(a)

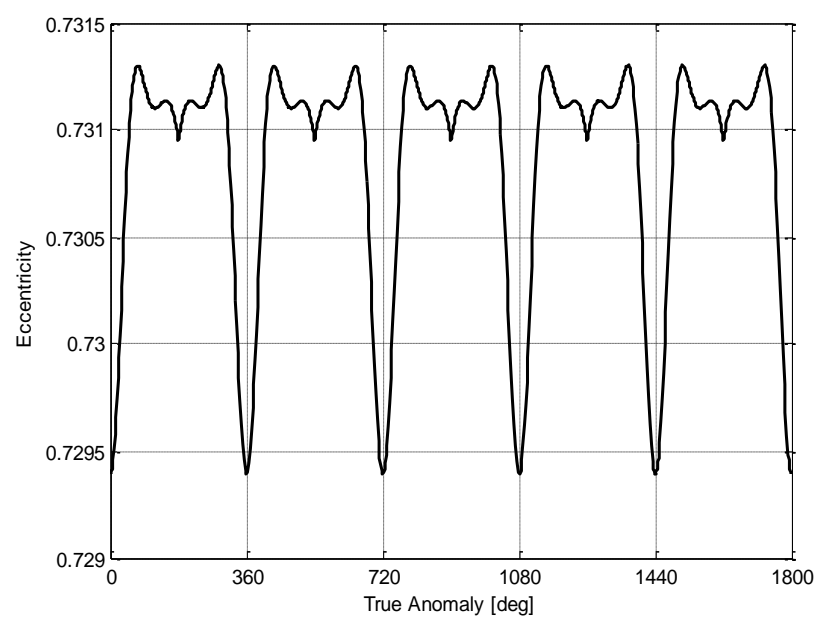

(b)

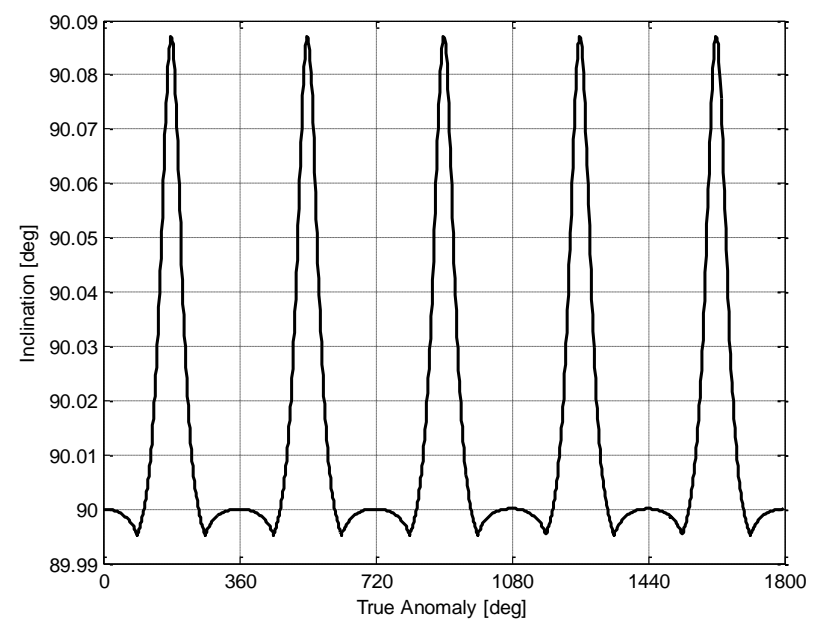

(c)

Fig. 7 Oscillation of orbital elements over five orbital revolutions: a) semimajor axis, b) eccentricity, and c) argument of perigee.

14

American Institute of Aeronautics and Astronautics 


\section{Conclusion}

The use of continuous low-thrust propulsion has been shown to enable highly elliptical sun-synchronous orbits around the Earth, termed sun-synchronous Taranis orbits. Continuous, constant acceleration is used to alter both the natural critical inclination of highly elliptical orbits to any inclination, and to maintain the sun-synchronous orbit condition. As such, a highly elliptical sun-synchronous orbit inclined at 90 deg can be enabled, allowing improved high-latitude imaging and simplified instrument design through simplification of the thermal environment. To enable a $12 \mathrm{~h}, 90$ deg inclination orbit a total acceleration of around $0.5 \mathrm{~mm} / \mathrm{s}^{2}$ is required, although this is significantly higher than the acceleration required to enable a highly elliptical orbit with an inclination of 90 deg without the sun-synchronous condition $\left(0.081 \mathrm{~mm} / \mathrm{s}^{2}\right)$, it is likely to be achievable using current or near term technology, for example the High Power Electric Propulsion Thruster.

\section{References}

1. Lazzara, M. A., Stearns, C. R., Staude, J. A., and Knuth, S. L. "10 Years of Antarctic Composite Images," 7th Conference on Polar Meteorology and Oceanography and Joint Symposium on High-Latitude Climate Variations. Vol. Paper 9.4, American Meteorological Society, Boston, MA, Hyannis, MA, 2003, 12-16 May 2003.

2. Pieri, D. C. "Virtual Polar Geostationary Satellite (VPGS): A Trade Study for the NASA Earth Science Technology Office.." Earth and Science Division Jet Propulsion Laboratory California Institute of technology Pasadena, CA.

3. "World Meteorological Organization vision for the GOS in 2025", Consulative Meetings on High-Level Policy on Satellite Matters Ninth Session, 2009.

4. Anderson, P., and Macdonald, M. "Extension of Highly Elliptical Earth Orbits using Continuous Low-Thrust Propulsion", Journal of Guidance, Control and Dynamics, Accepted November 2011.

5. Anderson, P., and Macdonald, M. "Static Highly Elliptical Orbits using Hybrid Low-Thrust Propulsion ", Journal of Guidance, Control and Dynamics, Submitted October 2011.

6. Anderson, P., and Macdonald, M. "Continuous Remote Sensing of Polar Regions from a Taranis Orbit Constellation", Journal of Atmospheric and Oceanic Technology, Submitted April 2012.

7. Macdonald, M., McKay, R. J., Vasile, M., and Bosquillon de Frescheville, F. "Extension of the Sun-synchronous Orbit", Journal of Guidance, Control and Dynamics Vol. 33, No. 6, 2010, pp. 1935-1940.

8. Fortescue, P., Stark, J., and Swinerd, G. Spacecraft Systems Engineering. England: John Wiley \& Sons Ltd. Chichester, West Sussex, England, 2003. pp 94.

9. Macdonald, M., and McInnes, C. R. "Analytical Control Laws for Planet-Centered Solar Sailing", Journal of Guidance, Control and Dynamics Vol. 28, No. 5, 2005, pp. 1038-1048.

10. Forster, J. E., Haag, T., Patterson, M., Williams, G. J., Sovey, J. S., Carpenter, C., Kamhawi, H., Malone, S., and Elliot, F. "The High Power Electric Propulsion (HiPEP) Ion Thruster", 40th Joint Propulsion Conference and Exhibit, 2004.

11. Randolph, T. M., and Polk, J. E. "An Overview of the Nuclear Electric Xenon Ion System (NEXIS) Activity.", 40th AIAA/ASME/SAE/ASEE Joint Propulsion Conference, 2004.

12. Walker, M. J. H., Ireland, B., and Owens, J. "A set of Modified Equinoctial Orbital Elements", Celestial Mechanics Vol. 36, 1985, pp. 409-419.

13. Dormand, J. R., and Prince, P. J. "A family of enbedded Runge-Kutta formulae", Journal of Computational and Applied Mathematics and Statistics Vol. 6, No. 1, 1980, pp. 19-26. 